\title{
Wolman Disease in Bulgarian Patients: Selective Genetic Screening in Two Presumable Endemic Regions
}

\author{
Angelina Mandadzhieva', Daniela Avdzhieva-Tzavella², Tihomir Todorov ${ }^{1}$, Savina Tincheva1, \\ Vanya Sinigerska ${ }^{3}$, Mariya Ivanova ${ }^{3,5}$, Alexey Savov3, Vanyo Mitev ${ }^{4}$, Albena Todorova ${ }^{1,4}$ \\ ${ }^{1}$ Genetic Medico-Diagnostic Laboratory “Genica”, Sofia, Bulgaria \\ ${ }^{2}$ Department of Genetics, University Pediatric Hospital, Medical University, Sofia, Bulgaria \\ ${ }^{3}$ National Genetic Laboratory, Department of Obstetrics and Gynecology, Faculty of Medicine, Medical University Sofia, Sofia, Bulgaria \\ ${ }^{4}$ Department of Medical Chemistry and Biochemistry, Medical University Sofia, Sofia, Bulgaria \\ ${ }^{5}$ Department of Analytical Chemistry, Faculty of Chemistry and Pharmacy, Sofia University “St. Kl. Ohridski”, Sofia, Bulgaria \\ Email: andji_m@abv.bg
}

How to cite this paper: Mandadzhieva, A., Avdzhieva-Tzavella, D., Todorov, T., Tincheva, S., Sinigerska, V., Ivanova, M., Savov, A., Mitev, V. and Todorova, A. (2017) Wolman Disease in Bulgarian Patients: Selective Genetic Screening in Two Presumable Endemic Regions. American Journal of Molecular Biology, 7, 169-175. https://doi.org/10.4236/ajmb.2017.74013

Received: June 2, 2017

Accepted: September 11, 2017

Published: September 14, 2017

Copyright (C) 2017 by authors and Scientific Research Publishing Inc. This work is licensed under the Creative Commons Attribution International License (CC BY 4.0).

http://creativecommons.org/licenses/by/4.0/ (c) (†) Open Access

\begin{abstract}
Wolman disease is a rare autosomal recessive disorder caused by mutations in the LIPA gene (10q23.31). The LIPA gene encodes lysosomal acid lipase (LAL), which plays a key role in hydrolysis of the cholesteryl esters and triglycerides. Two unrelated families from Bulgaria were referred for genetic testing with clinical diagnosis Wolman disease. Sanger sequencing of all coding exons and exon-intron boundaries of the LIPA gene was performed. The index patients were found to be homozygous for two different mutations in the LIPA gene: a missense mutation, c.260G > T, p.Gly87Val, which affects the enzyme active site and a splice-site change, c. $822+1 \mathrm{G}>\mathrm{A}$, which most probably destroys the enzyme polypeptide chain. These two completely different types of mutations along the LIPA gene resulted in a very similar phenotype involving liver, kidney, gastrointestinal, muscle and blood disturbances. As consanguinity is not typical for the Bulgarian population, a possible explanation of the homozygosity could be presence of endemic regions for given mutations. To check this hypothesis, selective screening for these mutations was performed in two presumable endemic regions in Bulgaria. Altogether, 100 newborns were screened for p.Gly87Val mutation and the detected carrier frequency was about $1 \%(1 / 100)$, while in the group of 100 newborns screened for the c. $822+1 \mathrm{G}>$ A mutation the detected carrier frequency was $2 \%$ $(2 / 100)$. The results indicate a high recurrence risk of Wolman disease in these particular Bulgarian regions of about 1:10000. These findings are from crucial importance for the inhabitants of the corresponding parts of Bulgaria. They may benefit from early genetic testing and adequate genetic counselling during family planning.
\end{abstract}




\section{Keywords}

Wolman Disease, LIPA Gene, Lysosomal Acid Lipase, Mutations, Selective Screening

\section{Introduction}

Wolman disease (WD MIM\# 278000) is a rare autosomal recessive disorder. It belongs to the group of lysosomal diseases and affects 1 in 100,000 newborn. The disease is associated with accumulation of cholesterol esters and enzyme deficit. Wolman disease is manifested with hepatosplenomegaly, abdominal distention, and adrenal calcification. Massive intracellular storage of both cholesteryl esters and triacylglycerols is observed in liver, adrenal gland, and intestine. The affected patients usually die within the first year of life [1]. Wolman disease is caused by mutations in the LIPA gene, localized on chromosome 10 (10q23.31) [2] [3]. The LIPA gene encodes an enzyme called lysosomal acid lipase (LAL) (EC: 3.1.1.13), which plays a key role in hydrolysis of the cholesteryl esters and triglycerides into free cholesterol and free fatty acids [4].

Recently, various types of therapies for lysosomal acid lipase deficiency have been reported [5] [6]. Some attempts are focused on hematopoietic stem cell transplantation and bone marrow transplantation, resulting in normalization of cholesterol levels [6] [7] [8]. Promising results are achieved in enzyme replacement therapy [6] [9] [10]. If not treated, the disease has fatal outcome. Therefore, it is very important for the patients and their families to be clinically diagnosed and genetically verified on time to benefit from therapy.

Here we report our results from molecular genetic diagnostics of the first unrelated Bulgarian families with Wolman disease. Both index patients were positive for LIPA gene mutations in homozygous state. As consanguinity is not typical for the Bulgarian population, a possible explanation of the homozygosity could be presence of endemic regions for given mutations. Based on our preliminary results, we performed selective newborn screening for particular LIPA gene mutations in two presumable endemic regions in Bulgaria.

\section{Materials and Methods}

Two unrelated families from Bulgarian origin were referred for genetic testing of the LIPA gene, because of the clinical diagnosis of Wolman disease. Two symptomatic unrelated patients and their asymptomatic parents were examined. Both families originated from two different geographical regions Kostenets (western Bulgaria) and Bansko (southwestern Bulgaria) (Figure 1).

Selective screening for particular mutations in the LIPA gene was performed in two presumable endemic regions: Kostenets (western Bulgaria) and Bansko (southwestern Bulgaria) (Figure 1). Altogether 200 samples (100 for each re- 
gion) were selected from Guthrie cards dry blood spots, collected for the aims of the national newborn screening programs (kindly provided by the National Genetic Laboratory, Sofia, Bulgaria). The study was approved by the Local Ethical Committee. Informed consent was signed by the parents prior to genetic testing.

\subsection{Genetic Testing}

DNA was extracted from peripheral blood by standard salting-out procedure. The screening for mutations in the LIPA gene was performed by Sanger sequencing of all coding exons and exon-intron boundaries, using specifically designed primers (the primer sequence is available upon request).

The cDNA reference sequence was based on information available from RefSeq (Accession number): Human LIPA, NM_000235. The sequencing reaction was performed by BigDye ${ }^{\oplus}$ Terminator cycle sequencing kit v.3.1 (Applied Biosystems, Foster City, CA, USA), which includes Thermo Sequenase II DNA polymerase and fluorescently labeled nucleotides. The electrophoretic separation was performed on ABI3130 Genetic Analyzer (Applied Biosystems, Foster City, CA, USA). The sequencing profiles were interpreted by the software Sequencing Analysis v5.1.1.

The selective screening for particular mutations in exons 4 and 7 of the LIPA gene was performed by direct PCR amplification of these exons on dry blood spots from Guthrie cards [11], followed by sequencing. The primers were designed to specifically amplify the coding part of the exon and exon-intron boundaries (Exon 4: F-5 '-GTGAGTACATCACTATGTCAATCTTTCA-3'/ R-5'-CCCCTCTCATACAACTTCAGAGTTA-3'; Exon 7:

F-5'-CCATTCTCTCCTGAGGCCAT-3'/

R-5'-CCTCTCAAATGAAAGACTCTTTAGAGTT-3').

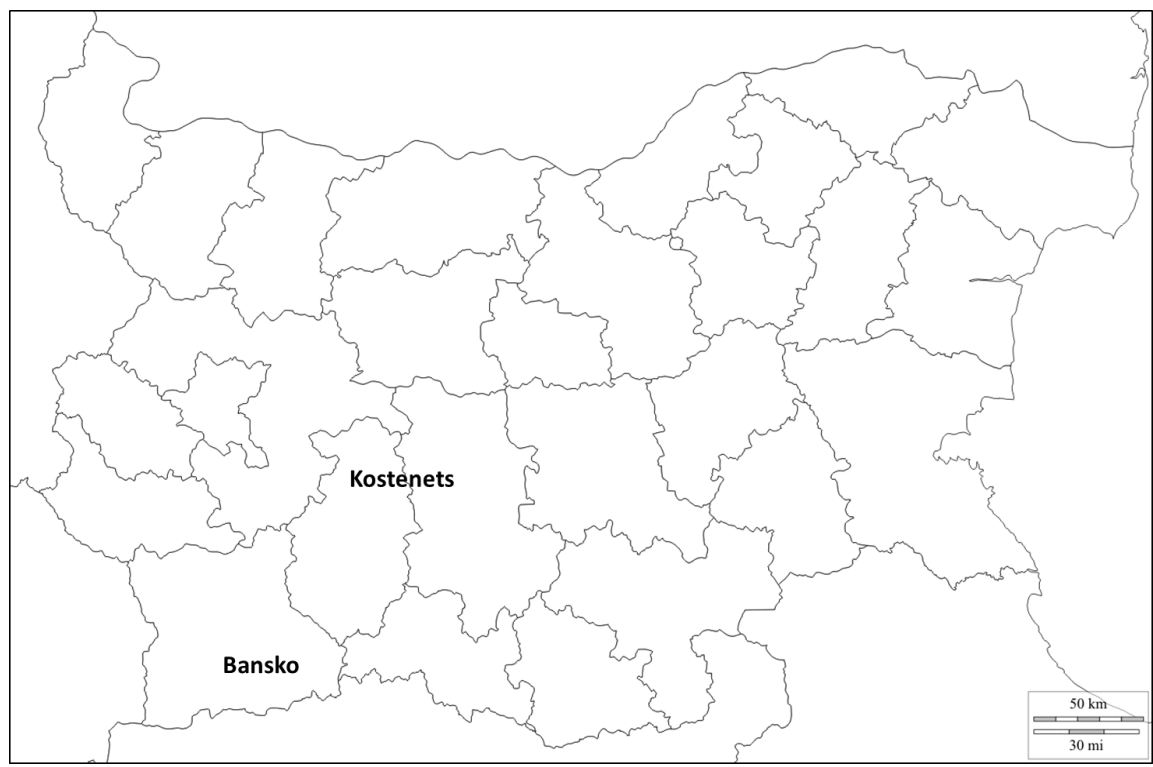

Figure 1. Presumable endemic regions: Kostenets and Bansko. 


\section{Compliance with Ethics Guidelines}

Angelina Mandadzhieva-Performed the genetic tests in the affected families and conducted the selective screening on dry blood spots; manuscript preparation.

Daniela Avdzhieva-Tzavella-Physician, who pointed the clinical diagnosis.

Tihomir Todorov-Planned the screening program, supervised the results from genetic testing and conducted the genetic verification of the diagnosis in both families; reporting of the results and patients counseling.

Savina Tincheva-Critical reading and writing of the manuscript.

Vanya Sinigerska, Marya Ivanova-Performed the metabolic analysis.

Alexey Savov-Head of the National Genetic Laboratory, provided access to the Guthrie cards.

Vanyo Mitev-Financial support of the study.

Albena Todorova-Head of the Genetic Medico-Diagnostic Laboratory "Genica", planning and design of the study, supervisor of the performed genetic and screening tests, critical reading and revision of the manuscript.

\section{Results and Discussion}

\subsection{Clinical Picture and LIPA Genotyping in Affected Families}

Two unrelated patients aged 3 and 5 months were referred for genetic testing of the LIPA gene due to the clinical symptoms compatible with Wolman disease. The parents claim not to have any consanguinity. No other affected relatives were reported. Both children expressed severe anemia, muscle hypotrophy and low weight gain. Liver problems like hepatosplenomegaly and hepatocytolysis were also present. Gastrointestinal dysfunction includes difficulties in bowel movement and appearance of watery stools with the disease progression. Kidneys are affected by adrenal glands calcification.

The enzyme levels of acid lipase were reduced- $-12.8 \mathrm{nmolMU} / \mathrm{h} / \mathrm{mg}$ and 38 $\mathrm{nmolMU} / \mathrm{h} / \mathrm{mg}$, at reference values 210-530.

DNA sequencing revealed two different mutations. The first mutation noted in our patient is a previously well-known disease-causing missense mutation, c.260G > T; p.Gly87Val in exon 4 of the LIPA gene [12] [13] [14]. The mutation substitutes the aminoacid glycine with valine. It has been previously reported that the valine branched chain interferes with the active-site serine residue and partly blocks the access to the presumed triglyceride-binding site of the lipase. The p.Gly87Val substitution results in abolished enzymatic activity of the lipase [1]. The mutation is tracked under the number NM_001127605.2 [15].

The second mutation is a splice-site change, c. $822+1 \mathrm{G}>\mathrm{A}$ in exon 7 of the LIPA gene, which affects donor consensus splice nucleotide guanine, resulting in altered mRNA splicing. This splice-site mutation was reported once in a patient from United States [16].

It is worth mentioning that both point mutations substitute the same purine nucleotide guanine $(G)$. A possible explanation for the higher mutability at $G$ 
nucleotide is that during replication guanine may undergo a tautomeric shift to its rare enol form, which pairs with thymine (T) [17]. In our first case $G$ is substituted with pyrimidine nucleotide $\mathrm{T}$, which results in transversion. The second substitution is transition of $\mathrm{G}$ to A nucleotide in the conserved donor splice-site. In general, transitions have been more frequently reported along the human genome than transversions [18] [19] [20]. Most probable explanation is the identical chemical structure of the bases involved in the transition events, which makes easier such a mistake to escape the proofreading activity of the polymerase, resulting in mutation fixation in the genome.

In respect to the genotype-phenotype correlations it is interesting to mention that genetic heterogeneity may be associated with very uniform disease manifestation. We found two completely different types of mutations along the LIPA gene: aminoacid substitution affecting the enzyme active-site and splicing defect, which most probably destroys the enzyme polypeptide chain, but both genetic variants resulted in very similar phenotype involving liver, kidney, gastrointestinal, muscle and blood disturbances.

Considering the high genetic heterogeneity of the Bulgarian population, it is extremely atypical to detect homozygous mutations in rare recessive conditions. A possible explanation could be consanguineous marriages, which are not typical for the Bulgarian population. On the other hand we may hypothesize higher mutation frequency of particular LIPA variants in defined geographic regions which may lead to homozygous affected offspring due to endogamous marriages. To check this hypothesis we further screened for these particular mutations newborn samples originating from the native regions of both patients.

\subsection{Mutation Screening in Presumable Endemic Regions}

Knowing the result from the molecular genetic testing of the first family (homozygous for the c.260G > T, p.Gly87Val mutation), we supposed the region of Kostenets could be endemic for this given mutation. We performed selective screening for the mutation p.Gly87Val in this part of the country. Altogether, 100 newborns originating from the corresponding region were tested for the p.Gly87Val mutation. Our results showed one heterozygous carrier, which represents a carrier frequency in this subpopulation of about $1 \%(1 / 100)$.

The second geographic region of interest in the context of Wolman disease turned out to be the region of Bansko situated in the southwest part of the country, where the second LIPA gene positive family originated from. We screened 100 newborns from the corresponding region, for the presence of $c .822+1 \mathrm{G}>\mathrm{A}$ mutation in the LIPA gene. Surprisingly, the molecular genetic testing in this region showed even higher carrier frequency of $2 \%(2 / 100)$. The results from the screening are provided in Table 1.

In conclusion, the performed selective screening for mutations in the LIPA gene aimed to search for particular gene variants p.Gly87Val and c.822+1G $>$ A in two targeted geographic regions-Kostenets and Bansko. The obtained results showed high carrier frequency of $1 \%$ and $2 \%$, respectively. Our results indicate a 
Table 1. Results from selective genetic screening.

\begin{tabular}{ccccc}
\hline $\boldsymbol{L I P A}$ gene & Guthrie samples Heterozygous carriers & Heterozygosity & Mutant allele frequency* \\
\hline Exon 4 & 100 & 1 & $1 \%$ & $0.5 \%$ \\
Exon 7 & 100 & 2 & $2 \%$ & $1 \%$ \\
\hline
\end{tabular}

**Both alleles of the LIPA gene for each of the 100 samples (altogether 200 alleles) were considered in the calculations.

high recurrence risk of Wolman disease in these particular Bulgarian regions of about 1:10000, which presumes the disease might be much more frequent than expected for the general population. These findings are from crucial importance for the inhabitants of the corresponding parts of Bulgaria. They may benefit from early genetic testing and adequate genetic counselling during family planning.

\section{Acknowledgements}

The dried blood spots from Guthrie cards of newborns were kindly provided by the National Genetic Laboratory, Sofia.

\section{Conflict of Interest}

The authors declare that they have no conflict of interest.

\section{References}

[1] Zschenker, O., Jung, N., Rethmeier, J., Trautwein, S., Hertel, S., Zeigler, M. and Ameis, D. (2001) Characterization of Lysosomal Acid Lipase Mutations in the Signal Peptide and Mature Polypeptide Region Causing Wolman Disease. Journal of Lipid Research, 42, 1033-1040.

[2] Aslanidis, C., Klima, H., Jackner, K. and Schmitz, G. (1994) Genomic Organization of the Human Lysosomal Acid Lipase Gene (LIPA). Genomics, 20, 329-331. https://doi.org/10.1006/geno.1994.1180

[3] Aslanidis, C., Ries, S., Fehringer, P., Buchler, C., Klima, H. and Schmitz, G. (1996) Genetic and Biochemical Evidence That CESD and Wolman Disease Are Distinguished by Residual Lysosomal Acid Lipase Activity. Genomics, 33, 85-93. https://doi.org/10.1006/geno.1996.0162

[4] Reiner, Z., Guardamagna, O., Nair, D., Soran, H., Hovingh, K., Bertolini, S., Jones, S., Coric, M., Calandra, S., Hamilton, J., Eagleton, T. and Ros, E. (2014) Lysosomal Acid Lipase Deficiency-An under Recognized Cause of Dyslipidaemia and Liver Dysfunction. Atherosclerosis, 235, 21-30. https://doi.org/10.1016/j.atherosclerosis.2014.04.003

[5] Xu, M., Motabar, O., Ferrer, M., Marugan, J., Zheng, W. and Ottinger, E. (2016) Disease Models for the Development of Therapies for Lysosomal Storage Diseases. Annals of the New York Academy of Sciences 2016, 1371, 15-29. https://doi.org/10.1111/nyas.13052

[6] Aguisanda, F., Thorne, N. and Zheng, W. (2017) Targeting Wolman Disease and Cholesteryl Ester Storage Disease: Disease Pathogenesis and Therapeutic Development. Current Chemical Genomics and Translational Medicine, 11, 1-18. https://doi.org/10.2174/2213988501711010001 
[7] Tolar, J., Petryk, A., Khan, K., Bjoraker, K.J., Jessurun, J., Dolan, M., Kivisto, T., Charnas, L., Shapiro, E.G. and Orchard, P.J. (2008) Long-Term Metabolic, Endocrine, and Neuropsychological Outcome of Hematopoietic Cell Transplantation for Wolman Disease. Bone Marrow Transplant, 43, 21-27. https://doi.org/10.1038/bmt.2008.273

[8] Krivit, W., Peters, C., Dusenbery, K., Ben-Yoseph, Y., Ramsay, N.K., Wagner, J.E. and Anderson, R. (2000) Wolman Disease Successfully Treated by Bone Marrow Transplantation. Bone Marrow Transplant, 26, 567-570. https://doi.org/10.1038/sj.bmt.1702557

[9] Barton, N.W., Brady, R.O., Dambrosia, J.M., Di Bisceglie, A.M., Doppelt, S.H., Hill, S.C., Mankin, H.J., Murray, G.J., Parker, R.I., Argoff, C.E., et al. (1991) Replacement Therapy for Inherited Enzyme Deficiencymacrophage-Targeted Glucocerebrosidase for Gauchers Disease. New England Journal of Medicine, 324, 1464-1470. https://doi.org/10.1056/NEJM199105233242104

[10] Braulke, T. and Bonifacino, J. (2009) Sorting of Lysosomal Proteins. Biochimica et Biophysica Acta, 1793, 605-614. https://doi.org/10.1016/j.bbamcr.2008.10.016

[11] Todorova, A., Ashikov, A., Beltcheva, O., Tournev, I. and Kremensky, I. (1999) C283Y Mutation and Other C-Terminal Nucleotide Changes in the G-Sarcoglycan Gene in the Bulgarian Gypsy Population. Human Mutation, 14, 40-44. https://doi.org/10.1002/(SICI)1098-1004(1999)14:1<40::AID-HUMU5>3.0.CO;2-R

[12] Pagani, F., Garcia, R., Pariyarath, R., Stuani, C., Gridelli, B., Paone, G. and Baralle, F. (1996) Espression of Lysosomal Acid Lipase Mutants Detected in Three Patients with Cholesteryl Ester Storage Disease. Human Molecular Genetics, 1611-1617. https://doi.org/10.1093/hmg/5.10.1611

[13] Pagani, F., Pariyarath, R., Garcia, R., Stuani, C., Burlina, A., Ruotolo, G., Rabusin, M. and Baralle, F. (1998) New Lysosomal Acid Lipase Gene Mutants Explain the Phenotype of Wolman Disease and Cholesteryl Ester Storage Disease. Journal of Lipid Research, 39, 1382-1388.

[14] Valles-Ayoub, Y., Esfandiarifard, S., Sinai, P., Khokher, Z., Kohan, M., Kahen, T. and Darvish, D. (2011) Wolman Disease (LIPA p.G87V) Genotype Frequency in People of Iranian-Jewish Ancestry. Genetic Testing and Molecular Biomarkers, 15, 395-398. https://doi.org/10.1089/gtmb.2010.0203

[15] https://www.ncbi.nlm.nih.gov/clinvar/variation/88770/

[16] Yang, Y., Fernandes, P.H., Miloslavskaya, I., Orchard, P., Schwarzenberg, S.J., Grimley, M., O’Brien, W.E. and Eng, C.M. (2009) Molecular Diagnosis of Wolman/CESD Disease: Mutation Detection and Genotype-Phenotype Correlations.

[17] Griffiths, A.J.F. (1999) The Molecular Basis of Mutation. Modern Genetic Analysis. New York

[18] Todorova, A. and Danieli, G.A. (1997) Large Majority of Single-Nucleotide Mutations along the Dystrophin Gene Can Be Explained by More than One Mechanism of Mutagenesis. Human Mutation, 9, 537-547. https://doi.org/10.1002/(SICI)1098-1004(1997)9:6<537::AID-HUMU7>3.0.CO;2-Z

[19] Li, W.-H., Wu, C.-I. and Luo, C.-C. (1984) Non-Randomness of Point Mutation as Reflected in Nucleotide Substitutions in Pseudogenes and Its Evolutionary Implications. Journal of Molecular Evolution, 27, 58-71. https://doi.org/10.1007/BF02100628

[20] Vogel, F. (1972) Non-Randomness of Base Replacement in Point Mutation. Journal of Molecular Evolution, 1, 334-367. https://doi.org/10.1007/BF01653962 